\title{
Comparison of EPI Distortion Correction Methods in Diffusion Tensor MRI Using a Novel Framework
}

\author{
M. Wu ${ }^{1,2}$, L.-C. Chang ${ }^{3}$, L. Walker ${ }^{1}$, H. Lemaitre ${ }^{4}$, A.S. Barnett ${ }^{4}$, \\ S. Marenco ${ }^{4}$, and C. Pierpaoli ${ }^{1}$ \\ ${ }^{1}$ National Institute of Child Health and Human Development, \\ National Institutes of Health, Bethesda, MD, USA \\ ${ }^{2}$ Dept. of Electrical and Computer Engineering, University of Pittsburgh, \\ Pittsburgh, PA, USA \\ ${ }^{3}$ Dept. of Electrical Engineering and Computer Science, Catholic University of America, \\ Washington, D.C., USA \\ ${ }^{4}$ National Institute of Mental Health, National Institutes of Health, Bethesda, MD, USA \\ cpla@nih.gov
}

\begin{abstract}
Diffusion weighted images (DWIs) are commonly acquired with Echo-planar imaging (EPI). B0 inhomogeneities affect EPI by producing spatially nonlinear image distortions. Several strategies have been proposed to correct EPI distortions including $\mathrm{B}_{0}$ field mapping $\left(\mathrm{B}_{0} \mathrm{M}\right)$ and image registration. In this study, an experimental framework is proposed to evaluation the performance of different EPI distortion correction methods in improving DTderived quantities. A deformable registration based method with mutual information metric and cubic B-spline modeled constrained deformation field (BSP) is proposed as an alternative when $\mathrm{B}_{0}$ mapping data are not available. BSP method is qualitatively and quantitatively compared to $\mathrm{B}_{0} \mathrm{M}$ method using the framework. Both methods can successful reduce EPI distortions and significantly improve the quality of DT-derived quantities. Overall, $\mathrm{B}_{0} \mathrm{M}$ was clearly superior in infratentorial regions including brainstem and cerebellum, as well as in the ventral areas of the temporal lobes while BSP was better in all rostral brain regions.
\end{abstract}

Keywords: DTI, EPI distortion correction, $\mathrm{B}_{0}$ field mapping, B-spline image registration.

\section{Introduction}

Clinical diffusion MR studies use almost exclusively single-shot echo-planar imaging (EPI) acquisitions. EPI is very sensitive to static magnetic field (B0) inhomogeneities that produce nonlinear geometric distortion primarily along the phase-encoding direction. Artifacts are most pronounced at air-tissue interfaces and are clearly evident in the ventral portions of the frontal and temporal lobes, particularly in areas near the sphenoid sinus and the temporal petrous bone. These artifacts become more severe at higher magnetic field strengths.

Different techniques have been reported to correct geometric distortions originating from $\mathrm{B}_{0}$ inhomogeneities in EPI images[1, 2, 3, 4]. The field map based method is 
a popular method, in which the $\mathrm{B}_{0}$ field inhomogeneity is computed from phase images acquired at different echo times[1,2]. Another field map based method proposed by Chen and Wyrwicz utilizes the field maps derived from multi-echo gradient-echo images, which avoids complicated phase unwrapping procedures, but is computationally more demanding [4, 3]. Another method proposed by Kybic, et al [5] uses image registration techniques for EPI spatial distortion correction. The distorted EPI image is registered to a corresponding anatomically correct MR image with an intensitybased least-squares similarity metric and the B-spline modeled deformation field. Or $\log$-intensity metric is used in the registration to improve the sensitivity in areas of low EPI signal [6].

The above EPI distortion correction methods remain virtually unused in the context of DTI, partly because there are no objective tests for establishing whether the improvement in the quality of the computed DTI data justifies the additional scan time and/or computational complexity. The main goals of this study are 1) to propose an experimental framework that would enable evaluating the performance of different EPI distortion correction methods in terms of improving the quality of DTI results; 2 ) to propose an image registration-based correction method as an alternative when $\mathrm{B}_{0}$ mapping data are not available; 3 ) to compare the image registration-based correction method and the standard $\mathrm{B}_{0}$ mapping correction method in their ability to improve the DT-MRI results.

\section{Methods}

\subsection{Methodological Framework for Evaluating the Performance of EPI Distortion Correction Strategies}

Given that EPI distortions occur in the phase encode direction, one can obtain DTderived quantities that are differently corrupted by EPI distortions, with datasets of diffusion-weighted images (DWIs) acquired with different phase encoding directions. A simple approach to evaluate the effect of EPI distortion on DT-MRI data is to measure, on a voxel by voxel basis, the variability of DT-derived quantities from these co-registered DWI datasets. Significantly higher variability will appear in regions that are most significantly affected by EPI distortions. An effective EPI distortion method should reduce the misalignment between these DTI datasets due to different EPI distortions and lead to a reduction in the variability of the DTI quantity.

In this study, four replicate DWI datasets were acquired for each subject as shown in Fig. 1, with acquisition protocols differing only in the phase-encoding direction: along the right-left (RL) and anterior-posterior (AP) direction with either negative (-) or positive $(+)$ sign of the phase encoding blips respectively. The positive or negative blips will result in either compression or expansion distortion of the image along the phase-encoding direction.

The local variability of DT-MRI derived quantities (for example, fractional anisotropy (FA)) was assessed by the standard deviation map (SD) of the FA maps computed from the four DWI datasets, as in equation (1)

$$
S D_{F A}=\operatorname{std}\left(F A_{R L+}, F A_{R L_{-}}, F A_{A P_{+}}, F A_{A P_{-}}\right)
$$




\subsection{Subjects and Imaging Methods}

Five young subjects ( 2 males; mean age $=35.95$, range $24-48$ years old $)$ participated in this study. Written informed consent was obtained. Data were acquired on a 1.5 Tesla scanner (GE Medical Systems, Milwaukee, WI) equipped with an 8-channel RF coil. Diffusion weighted images were acquired with a single-shot spin-echo EPI sequence $(\mathrm{FOV}=24 \times 24 \mathrm{~cm}$, slice thickness $=2.5 \mathrm{~mm}$, no gap, matrix $=96 \times 96,60$ axial slices). Each DTI dataset consisted of 2 images with $b=0 \mathrm{~s} / \mathrm{mm}^{2}$ and 12 images with $\mathrm{b}=1100 \mathrm{~s} / \mathrm{mm}^{2}$ with different orientations of diffusion sensitization. An undistorted T2 weighted scan (T2WFSE) was acquired with a fast spin echo sequence $(\mathrm{FOV}=24 \mathrm{~cm}$, $\mathrm{TR}=9000 \mathrm{~ms}, \mathrm{TE}=81.504$, matrix $=256 \times 256$, slice thickness $=2.5 \mathrm{~mm}$, no gap, number of slice $=60$ ). For $\mathrm{B}_{0}$ field mapping, two gradient-echo images with different echo times $(\mathrm{TE}=7$ and $11.5 \mathrm{~ms}, \mathrm{FOV}=22 \mathrm{~cm}, \mathrm{TR}=1300 \mathrm{~ms}$, slice thickness $=2.0 \mathrm{~mm})$ were collected.

\subsection{EPI Distortion Correction Methods}

Image Registration based correction method: The distorted EPI image (the first $\mathrm{b}=0$ image in the DWI dataset) was registered to the anatomically undistorted T2WFSE image using a deformable registration algorithm with mutual information (MI) metric and cubic B-spline modeled deformation field. In the remainder of the paper we refer to this approach as the BSP method. The deformation was constrained to be only in the phase-encoding direction. The mutual-information based metric enables the registration of images with different modalities such as T1-weighted or T2-weighted images and the anatomically undistorted T2WFSE image is used as the target image for EPI correction in this study. The registration algorithm was implemented in C++ using the registration library in the Insight Segmentation and Registration Toolkit (ITK, www.itk.org)[7].

Field map based method: The $\mathrm{B}_{0}$ field map was calculated as the phase difference between the two gradient echo images divided by the echo time difference using tools from Oxford Center for Functional Magnetic Resonance Imaging of the Brain software library (FSL, www.fmrib.ox.ac.uk/). The complex field map was first phaseunwrapped using Phase Region Expanding Labeller for Unwrapping Discrete Estimates (PRELUDE)[8] and then was registered to the EPI data via rigid body registration, which was then applied to the EPI images using FMRIB's Utility for Geometrically Unwarping EPIs (FUGUE) in the phase-encoding direction for distortion correction. In the reminder of the paper we refer to this approach as the $\mathrm{B}_{0} \mathrm{M}$ method.

Prior to tensor computation, DWIs were processed with a pipeline consisting of the following steps: 1) the T2WFSE image was aligned to the anterior commissureposterior commissure (AC-PC) plane [9] and used as the target image (structural template) for all 4 DWI data; 2) both the structural template and the distorted EPI image (the first $b=0$ image in the DWI dataset) were noise padded to eliminate spurious boundaries; 3) All DWIs were corrected for motion and eddy current distortion [10]; 4) the structural template image was registered to the first $b=0$ image in the DWI dataset with an MI based rigid body registration to create a structural target for EPI distortion correction; 5) The deformation field for EPI correction was calculated in the 
native DWI space using $\mathrm{BSP}$ and $\mathrm{B}_{0} \mathrm{M}$ respectively; 6) For each method, the computed deformation field was applied to all DWIs in their native space; 7) DWIs were then reoriented to the structural template space, with proper rotation of the b-matrix [10]. Following these correction steps the diffusion tensor was computed using non-linear fitting [11] and its derived quantities including FA and Trace (TR) were calculated.

\subsection{Evaluation of Results and Statistical Analysis}

The effects of EPI distortion on the SD maps were first evaluated by visual inspection. For a quantitative assessment, the Wilcoxon signed-rank test was used on each subject for the comparison of the SD maps from different methods. The median of the SD map is computed as a global measurement of the performance of the corresponding EPI distortion correction method for each subject. The EPI correction method with the lowest median SD has the best overall performance. Population statistics were performed by paired t-test analysis of the median SD values across 5 subjects.

In order to inspect the spatial distribution of the effects in the population of five subjects, a hierarchical coarse-to-fine image registration pipeline [12] was used to transform each subject's structural image to the standard Montreal Neurological Institute (MNI) template colin27 [13]. The computed transformation was then used to spatially normalize the corresponding SD maps from DWI datasets with $\mathrm{BSP}, \mathrm{B}_{0} \mathrm{M}$ correction, and without EPI correction (NoC) into the template colin27 space. An average SD map for FA and TR across 5 subjects was generated for each method (represented as $S D_{A v g, F A, B S P}, S D_{A v g, F A, B_{0} M}$ and $S D_{A v g, F A, N o C}$ and respectively).

\section{Results}

Fig. 1 shows the distorted EPI images $(b=0)$ from 4 DWI datasets with different phase-encoding directions as well as the undistorted target image. The distortions are more pronounced in regions close to tissue-air interfaces such as the frontal poles and the temporal lobes near the petrous bone.

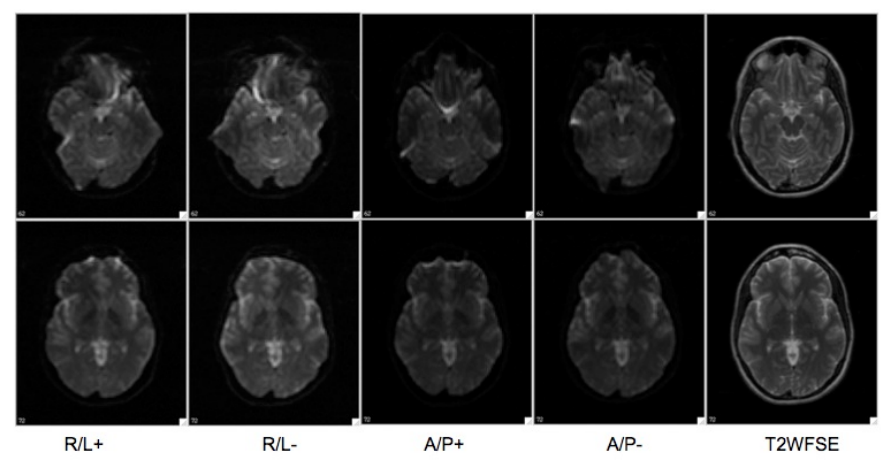

Fig. 1. The EPI distorted image slices with different phase encoding directions and the anatomically correct T2-FSE image slices of the same subject are shown here 
Fig. 2 shows a magnified view of an axial slice in a region of the brain relatively remote from sources of susceptibility variation. Arrows are marked at the same locations in all images to facilitate appreciating the degree of local distortion. Even at this location the uncorrected EPI image shows an appreciable amount of distortion, and both correction methods ( $\mathrm{BSP}$ and $\mathrm{B}_{0} \mathrm{M}$ ) have successfully reduced the distortion.

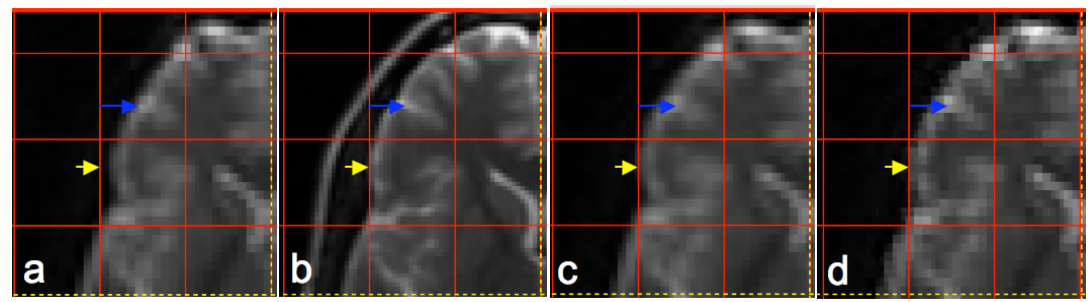

Fig. 2. An example of the distortion correction: (a) distorted RL- b=0 EPI image, (b) undistorted T2WFSE, (c) image corrected with BSP and (d) image corrected with $\mathrm{B}_{0} \mathrm{M}$

Mean and SD images from a representative subject were computed and displayed for FA (Fig. 3) and TR (Fig. 4). As expected for both FA and TR, higher SD values are visible in regions most affected by magnetic susceptibility variations such as tempral and frontal regions including the corpus callosum. SD decreases after EPI distortion correction with both methods. TR SD images demonstrate high variability at the top of the brain, and in periventricular regions.

In Fig. 5, the average performance of BSP across the population is compared to that of $\mathrm{B}_{0} \mathrm{M}$ by computing the variability difference map $S T D_{A v g, B_{0} M}-S T D_{A v g, B S P}$. The dark regions in Fig. 5(b)\&(c) indicate that, for EPI distortion correction, the $\mathrm{B}_{0} \mathrm{M}$ method performs better than the BSP method, and the bright regions are where BSP

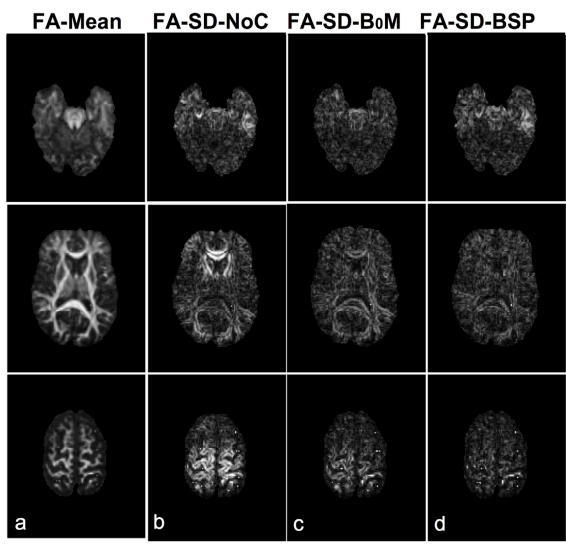

Fig. 3. Left: Results from a representative subject. (a) mean FA image with BSP correction (display range [0 0.95]), (b, c, and d) SD maps (display range [0 0.3]) of FA images from DTI datasets with no EPI correction (NoC), $\mathrm{B}_{0} \mathrm{M}$ correction, and BSP correction respectively. 


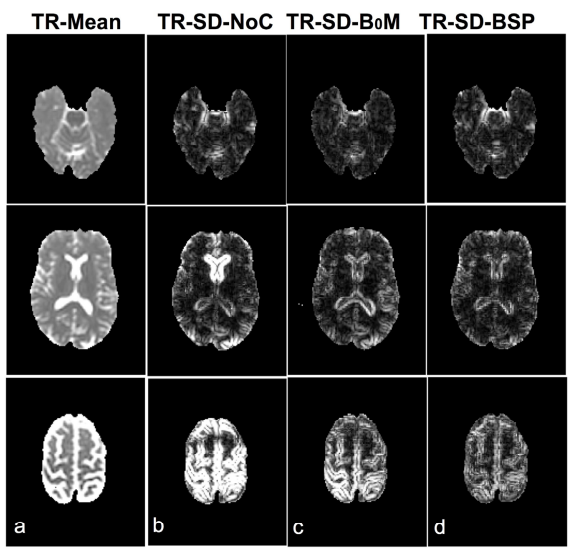

Fig. 4. Right: Results on a representative subject: (a) mean TR image with BSP correction with display range [0 $5000 \mu \mathrm{m}^{2} / \mathrm{s}$ ], (b, c, and d) SD maps (display range [0 $\left.1000 \mu \mathrm{m}^{2} / \mathrm{s}\right]$ ) of TR images from DTI datasets with no EPI correction (NoC), $\mathrm{B}_{0} \mathrm{M}$ correction, and BSP correction respectively

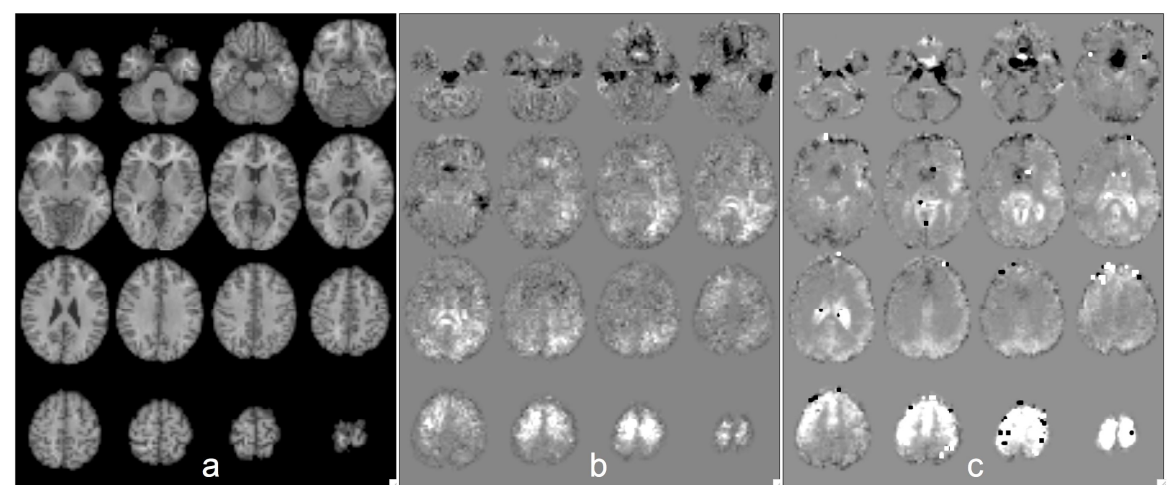

Fig. 5. Performance comparison of $\mathrm{BSP}$ and $\mathrm{B}_{0} \mathrm{M}$ across population. (a) $\mathrm{MNI}$ colin27; difference in the variability of (b) FA ( $S D_{A v g, F A, B_{0} M}-S D_{A v g, F A, B S P}$ ) with the display range of [-0.03 0.03], and (c) TR $\left(S D_{A v g, T R, B_{0} M}-S D_{A v g, T R, B S P}\right)$ with range [-200 $\left.200 \mathrm{~mm}^{2} / \mathrm{s}\right]$.

performs better than $\mathrm{B}_{0} \mathrm{M}$. BSP consistently provides better correction in rostral regions (e.g. corpus callosum, top of the brain), while $\mathrm{B}_{0} \mathrm{M}$ performs better at the base of the brain, including temporal lobes, brainstem, and cerebellum.

The median SD value of FA and TR from all brain voxels in each of the 5 subjects with or without EPI correction (BSP, $\mathrm{B}_{0} \mathrm{M}$, and NoC) is shown in Table 1 .

The percent change in median $\mathrm{SD}$ with different EPI distortion correction strategies is also calculated (e.g. $\mathrm{B}_{0} \mathrm{M}$ vs. $\left.\mathrm{NoC}=100^{*}\left(\mathrm{~B}_{0} \mathrm{M}-\mathrm{NoC}\right) / \mathrm{NoC}\right)$. Compared to the $\mathrm{SD}$ values with no EPI correction, on average, $\mathrm{B}_{0} \mathrm{M}$ reduced the median SD for $\mathrm{FA}$ by $12.8 \%$ and the median SD for TR by $16.2 \%$. BSP lowered the average median SDs of FA by $16.3 \%$ and TR by $23.2 \%$. For all 5 subjects, BSP consistently provided lower 
Table 1. Comparison of median values of SD maps (FA/TR) with or without EPI distortion correction

\begin{tabular}{|c|c|c|c|c|c|c|c|}
\hline \multirow{3}{*}{ Subject } & & \multicolumn{3}{|c|}{ Median SD } & \multicolumn{3}{|c|}{ Percent Change $\%$} \\
\hline & & & & & $\mathrm{B}_{0} \mathrm{M}$ & BSP & BSP \\
\hline & & 1 NoC & & DST & & & \\
\hline \multirow{5}{*}{ FA } & 1 & 0.0402 & 0.0347 & 0.0334 & -13.7 & -17.1 & -4.0 \\
\hline & 2 & 0.0413 & 0.0373 & 0.0365 & -9.5 & -11.7 & -2.5 \\
\hline & 3 & 0.0431 & 0.0373 & 0.0365 & -13.6 & -15.4 & -2.1 \\
\hline & 4 & 0.0435 & 0.0366 & 0.0347 & -15.9 & -20.3 & -5.5 \\
\hline & 5 & 0.0445 & 0.0397 & 0.0368 & -11.4 & -17.2 & -7.0 \\
\hline \multirow{5}{*}{ TR } & 1 & 186.9 & 153.4 & 141.3 & -17.9 & -24.4 & -8.5 \\
\hline & 2 & 136.2 & 122.0 & 115.8 & -10.4 & -15.0 & -5.4 \\
\hline & 3 & 139.4 & 117.1 & 110.8 & -16.0 & -20.5 & -5.7 \\
\hline & 4 & 206.5 & 163.9 & 143.5 & -20.6 & -30.5 & -14.2 \\
\hline & 5 & 172.5 & 144.7 & 128.2 & -16.2 & -25.7 & -12.8 \\
\hline
\end{tabular}

median SD than $\mathrm{B}_{0} \mathrm{M}$ for both FA (range [2.0\% 6.9\%]) and TR (range [5.3\% 14.2\%]). These results were confirmed by the voxel-wise Wilcoxon signed-rank tests, which showed that for all 5 subjects, $\mathrm{B}_{0} \mathrm{M}$ and BSP resulted in significantly lower SD values (FA or TR) than NoC and SD values from BSP were significantly lower than that of $\mathrm{B}_{0} \mathrm{M}$.

Paired two-tailed t-tests show that, across the population, median SD from BSP or $\mathrm{B}_{0} \mathrm{M}$ are significantly lower than NoC at $\mathrm{p}<0.0005$ (FA) and $\mathrm{p}<0.006$ (TR), and the median SD from BSP is significantly lower than $\mathrm{B}_{0} \mathrm{M}$ at $\mathrm{p}<0.012$ (FA and TR).

\section{Discussion}

One goal of this work was to propose a framework that would enable evaluating the performance of different EPI distortion correction methods in the context of DTI. We found that our relatively simple approach of calculating SD maps of the DTI quantities of interest for datasets with different phase-encoding directions can be effectively used for this purpose. The best EPI distortion correction strategy would produce an SD map with the lowest overall magnitude. Moreover, by analyzing SD maps, one can assess potential regional effects of the different correction algorithms. The SD maps of both FA and TR were computed and evaluated, since different DTI quantities may show different local sensitivity to the same EPI distortion. In fact, the spatial pattern of increased variability is not identical for FA and TR. For example, TR shows a clear pattern of increased variability in periventricular regions that is not present in FA.

For uncorrected data, the effects of misalignment were very noticeable in the genu of the corpus callosum for both FA and TR, ventral portions of the frontal lobe in proximity of the frontal sinuses mostly for TR, and ventral portions of the temporal lobe near the petrous bone for FA. These results are consistent with the fact that the 
largest distortions are to be expected in regions where $\mathrm{B}_{0}$ inhomogeneities are most pronounced.

We found high SD values in regions at the top of the brain, a region that is not normally associated with EPI distortions because it is distant from sinuses and other air-filled cavities. The effects of misalignment on the SD of both FA and TR could be magnified in apical brain regions because of the presence of sharp boundaries between CSF, gray matter, and white matter.

Both EPI distortion correction methods, $\mathrm{BSP}$ and $\mathrm{B}_{0} \mathrm{M}$, were effective in reducing the EPI geometric distortions and significantly improved the reproducibility of DTderived quantities. Interestingly, BSP showed an overall better performance than $\mathrm{B}_{0} \mathrm{M}$, although the two methods showed different performance in different regions. $\mathrm{B}_{0} \mathrm{M}$ was clearly superior in infratentorial regions including brainstem and cerebellum, as well as in the ventral areas of the temporal lobes. BSP was better than $\mathrm{B}_{0} \mathrm{M}$ in all rostral brain regions. While it is easy to understand why $\mathrm{B}_{0} \mathrm{M}$ performs better than BSP at the base of the brain where the susceptibility gradients are steep, it is more difficult to explain why $\mathrm{B}_{0} \mathrm{M}$ does not do as well as BSP in the rostral areas of the brain. One could hypothesize that the distortions at the top of the brain are not completely caused by $\mathrm{B}_{0}$ inhomogeneities but we have been unable to identify a definitive cause.

The most important practical conclusion of our work is that in the absence of $\mathrm{B}_{0}$ field data, successful EPI distortion correction of DTI data can be performed with a B-spline image registration approach using an undistorted T2-weighted image as the registration target.

In future work, one may combine these two methods to reach a more accurate deformation field for the whole brain in EPI distortion correction, such as using the deformation field from $\mathrm{B}_{0} \mathrm{M}$ as an initialization to correct regions at the base of brain with severe signal loss, and the BSP method as a finer step to improve the distortion correction in supratentorial brain regions.

\section{Reference}

1. Weisskoff, R.M., Davis, T.L.: Correcting gross distortion on echo planar images. In: Proceedings of the SMRM 11th Annual Meeting, p. 4515 (1992)

2. Jezzard, P., Balaban, R.S.: Correction for geometrical distortion in echo planar images from bo field variations. Magn. Reson. Med. 34, 65-73 (1995)

3. Chen, N.K., Wyrwicz, A.M.: Optimized distortion correction technique for echo planar imaging. Magn. Reson. Med. 45(3), 525-528 (2001)

4. Chen, N.K., Wyrwicz, A.M.: Correction for epi distortions using multi-echo gradient-echo imaging. Magn. Reson. Med. 41(6), 1206-1213 (1999)

5. Kybic, J., Thevenaz, P., Nirkko, A., Unser, M.: Unwarping of unidirectionally distorted epi images. IEEE Trans. Med. Imaging 19(2), 80-93 (2000)

6. Studholme, C., Constable, R.T., Duncan, J.S.: Accurate alignment of functional epi data to anatomical mri using a physics-based distortion model. IEEE Trans. Med. Imaging 19(11), 1115-1127 (2000)

7. Yoo, T.S.: Insight into images: Principles and practice for segmentation, registration, and image analysis. AK Peters Ltd., Wellesey (2004) 
8. Jenkinson, M.: Fast, automated, n-dimensional phase-unwrapping algorithm. Magn. Reson. Med. 49(1), 193-197 (2003)

9. Bazin, P.L., Cuzzocreo, J.L., Yassa, M.A., Gandler, W., McAuliffe, M.J., Bassett, S.S., Pham, D.L.: Volumetric neuroimage analysis extensions for the mipav software package. J. Neurosci. Methods 165(1), 111-121 (2007)

10. Rohde, G.K., Barnett, A.S., Basser, P.J., Marenco, S., Pierpaoli, C.: Comprehensive approach for correction of motion and distortion in diffusion-weighted MRI. Magn. Reson. Med. 51(1), 103-114 (2004)

11. Basser, P.J., Mattiello, J., LeBihan, D.: Estimation of the effective self-diffusion tensor from the nmr spin echo. J. Magn. Reson B 103(3), 247-254 (1994)

12. Wu, M., Carmichael, O., Lopez-Garcia, P., Carter, C.S., Aizenstein, H.J.: Quantitative comparison of air, spm, and the fully deformable model for atlas-based segmentation of functional and structural mr images. Hum. Brain Mapp. 27(9), 747-754 (2006)

13. Holmes, C.J., Hoge, R., Collins, L., Woods, R., Toga, A.W., Evans, A.C.: Enhancement of $\mathrm{mr}$ images using registration for signal averaging. Journal of Computer Assisted Tomography 22(2), 324-333 (1998) 